\title{
Flux density variations of radio sources in M82 over the last 3 decades
}

\author{
M. A. Gendre* \\ Jodrell Bank Centre for Astrophysics, University of Manchester, UK

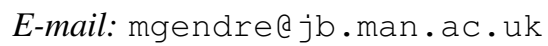 \\ D. M. Fenech \\ Department of Physics and Astronomy, University College London, UK

\section{R. J. Beswick} \\ Jodrell Bank Centre for Astrophysics, University of Manchester, UK
}

\section{T. W. B. Muxlow}

Jodrell Bank Centre for Astrophysics, University of Manchester, UK

\section{K. Argo}

Netherlands Institute for Radio Astronomy (ASTRON), Dwingeloo, The Netherlands

\begin{abstract}
This paper presents results of monitoring sessions on the starburst galaxy M82, obtained in 20092010 with the Multi-Element Radio-Linked Interferometer Network (MERLIN) at $5 \mathrm{GHz}$ and $e$-MERLIN at $6 \mathrm{GHz}$. Combining several $5 \mathrm{GHz}$ MERLIN epochs to form a map with a noise level of $33.0 \mu \mathrm{Jy} / \mathrm{beam}, 52$ discrete sources, mostly supernova remnants (SNRs) and HII regions, have been identified. These include three objects which were not detected in the $5 \mathrm{GHz}$ MERLIN observations conducted in 2002: supernova SN2008iz, the transient source 43.78+59.3, and a new supernova remnant shell, 44.28+61.1. Flux density variations, in the long (1981 to 2010), medium (2002 to 2010) and short (2009 to 2010) term, are investigated. We find that flux densities of SNRs in M82 stay constant in most ( $95 \%$ ) of the sample. In addition, aside from SN2008iz and the well-known variable source $41.95+57.5$, two sources, $43.18+58.2$ and 44.01+59.6, display hints of short and medium term variations over the period 2009-2010. Such flux density variations might be due to changes in the circumstellar and interstellar medium in which the shocks travel since these sources are among the most compact SNRs in M82.
\end{abstract}

11th European VLBI Network Symposium \& Users Meeting,

October 9-12, 2012

Bordeaux, France

${ }^{*}$ Speaker. 


\section{Observations}

The 2009 monitoring campaign of M82 consisted of seven wide-field MERLIN observations at a frequency of $4.994 \mathrm{GHz}$ observed between May 2009 and April 2010. These were made using parallel hands of circular polarisation, and were correlated with a total bandwidth of $16 \mathrm{MHz}$ divided into 32 channels. Across all epochs combined a total on-source integration time of 286.5 hours was used. Each observing epoch was reduced and analysed individually and a deep exposure map was produced by combining all of these data. In addition to these, a single $e$-MERLIN observation was included in this study. This observation was made in December 2010 as part of the $e$-MERLIN commissioning programme and used a total bandwidth of $512 \mathrm{MHz}$ with a median frequency of $6.26 \mathrm{GHz}$. It was correlated with four individual sub-bands each divided into 512 frequency channels. This $e$-MERLIN observation was observed prior to the installation of the new $e$-MERLIN wide-band IF system in spring 2011 and subsequently only one hand of polarisation was used and the data displayed reduced sensitivity in parts of the observing band.

\section{Source variability}

To improve our analysis of flux density variations, archival $5 \mathrm{GHz}$ MERLIN data from 2002 [四] and 2005 [四], as well as VLA data taken over an 11-year period starting in 1981 [囫] were added to the light curves. A systematic shift of $\sim 1.07$ mJy between the VLA and MERLIN data was removed from the VLA data. This difference in the overall flux density of each source is due to the larger beam size of the VLA observations, which are sensitive to more diffuse matter than our MERLIN data. Changes in the flux density of the detected sources were studied in the short, medium and long term (over the 2009-2010, 2002-2010 and 1981-2010 periods, respectively). A straight line $S(t)$ (with $t$ in months for short-term and years for medium- and long-term variations) was fitted through every light curve and the slope was used to determine which sources displayed consistent flux density variations. The distribution of slopes was fitted with a Gaussian model (see Fig. W). Outliers indicate sources with noticeable variability (SN2008iz and the variable source $41.95+57.5$, which show extreme variations, are not represented in these plots).

A total of six sources have shown flux density changes (i.e. with rates of change outside of a $2.5 \sigma$ limit) in the short, medium or long term, including $41.95+57.5$, SN2008iz and the transient source $43.78+59.3$. Two of the three other varying sources $(43.18+58.2$ and $44.01+59.6)$ have small angular sizes, and are some of the most compact supernova remnants (SNRs) in M82 after $41.95+57.5$ (Fig. \). The third source showing flux density changes, 46.52+63.9, shows only medium term variability. From its light curves in Fig. \, the variations can be explained by an anomalously low flux density value in 2002 . The source is then excluded from the varying sample.

SNRs in M82 are expected to be the result of core-collapse supernova events with a massive, typically red supergiant (RSG), progenitor star [5]. At the time of the supernova explosion, there will be a complex circumstellar environment consisting of a slow-moving dense RSG wind followed by a wind-blown bubble of almost constant low density produced by the main-sequence low-density, high-velocity wind. It is generally accepted that following the evolution through the wind-blown bubble, there will be a marked peak in flux density as the supernova ejecta travels from the low-density wind-blown bubble into the higher density ISM (especially in the case of M82). 

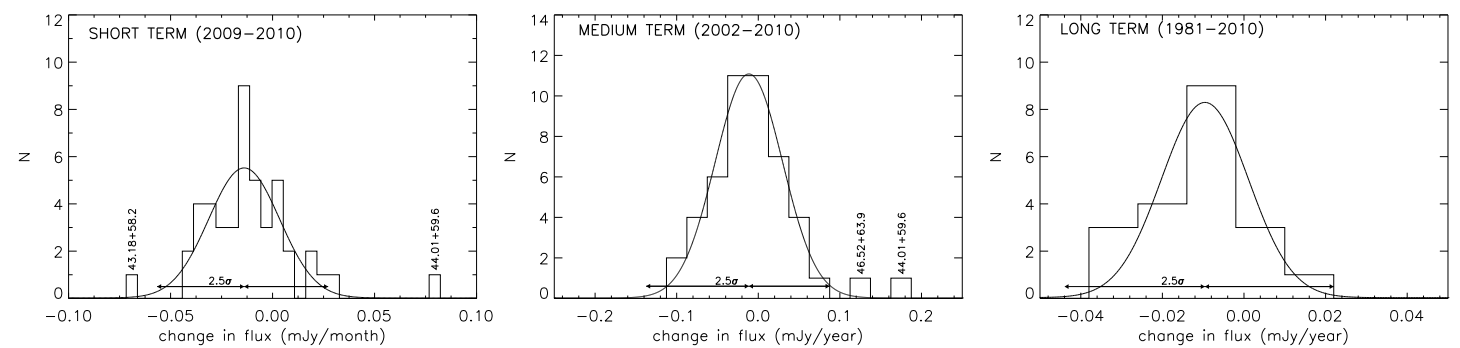

Figure 1: Short (2009-2010 period, left), medium (2002-2010 period, centre) and long (1981-2010 period, right) term variability. Names correspond to sources with rates of change $2.5 \sigma$ outside of the distribution. SN2008iz and the variable source $41.95+57.5$ lie well outside the range of this plot and are not represented.

This will primarily be caused by the production of a thin-dense shell at the edge of the wind-blown bubble. There will however, also be a subsequent decline in flux density as the ejecta traverses this shell and emerges into the ISM, traditionally considered as the transitional phase from a supernova to a supernova remnant. It is possible that this could provide an explanation for the observed fluxdensity decay. Continued regular monitoring of these sources will give us more insights into their behaviour. It is now possible to carry this out with new sensitive instruments such as $e$-MERLIN and the EVLA, and we expect to get a more complete view of the evolution of SNRs in M82.

\section{41.95+57.5 - A known variable source}

This source has shown a continued decrease in flux density of $\sim 8.8 \% / \mathrm{yr}$ since its first observation in 1965 [Q]. This decay rate would imply that, at birth, the source would have had a flux density of $\sim 30 \mathrm{Jy}$. These two facts (continuous decay and high flux density at birth) suggest that $41.95+57.5$ is likely to be an exotic event and several suggestions have been made as to its nature, including the possibility that it may have been an off-axis gamma-ray burst [ 8$]$ ].

As seen in Fig. [3], 41.95+57.5 still shows a steady decrease in flux density. However, the rate of this decrease was reduced from $8.8 \% / y r$ in 1981 to $6.4 \% / y r$ in 2010, when fitting over 1993-2010. VLBI observations in November 1998 and February 2001 show $41.95+57.5$ to be expanding at a rate of $\sim 2000 \pm 500 \mathrm{~km} \mathrm{~s}^{-1}$. However, further VLBI data from 2005 show that the source apparent radius may have decreased between 2001 and 2005 (30.4 mas in 2001 and 26.85 mas in 2005).

The unusually luminous radio supernova $1986 \mathrm{~J}$ in NGC891 and 41.95+57.5 show some similarities. Both sources display an asymmetric radio structure on milliarcsecond scales, modest expansion velocities, relatively high initial radio luminosities and a power-law flux density decay [3]. The possible decrease in apparent angular size and the small deviation from the historical flux density decline recently observed for $41.95+57.5$ are consistent with the potential appearance of a new higher frequency central component within the shell. As such, $41.95+57.5$ may be undergoing an evolution analogous to that observed in SN1986J, albeit in a much older remnant. Unfortunately, the MERLIN and $e$-MERLIN observations presented here do not have adequate angular resolution to image in detail the structure of this source and, as yet, higher frequency ( $>1.6 \mathrm{GHz})$ VLBI observations have not confirmed this hypothesis. Further analysis of the flux density variations of $41.95+57.5$ and SN1986J, both in time and frequency, are needed to assess more completely the similarity between the two sources. 

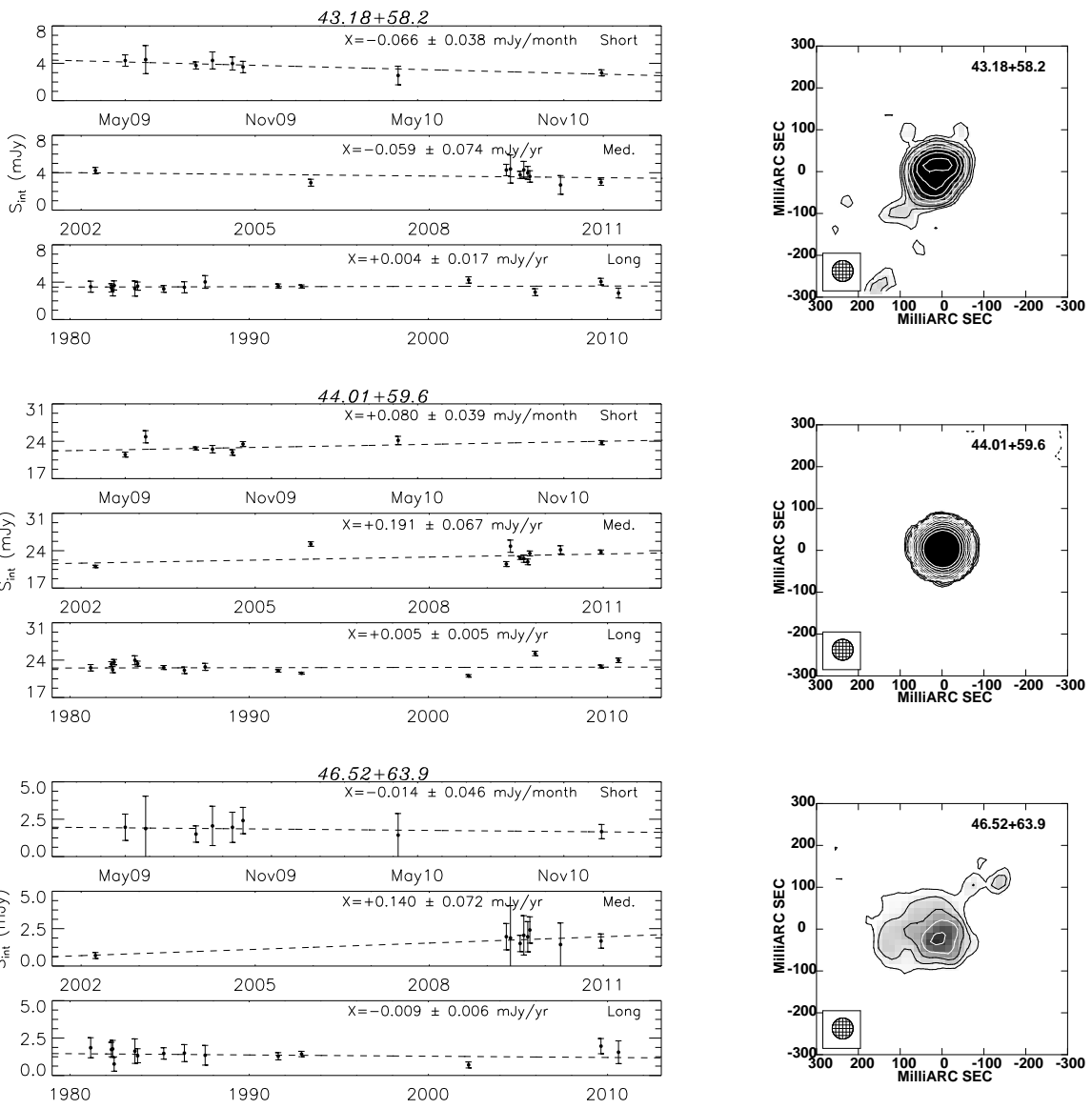

Figure 2: Left: short-, medium- and long-term light curves over the periods 2009-2010 (top), 2002-2010 (centre) and 1981-2010 (bottom, where the 1981-1991 data are from Kronberg et al. [䧃]), for sources showing noticeable changes in flux density, aside from $41.95+57.5$ and SN2008iz. For long-term variations, flux density measurements were yearly averaged in 2009 and 2010. A straight line $S(t)=S_{0}+X t$ (shown as a dashed line) was fitted to each light curve, where $X$ characterises the variation in $\mathrm{mJy} / \mathrm{month}$ and $\mathrm{mJy} / \mathrm{yr}$ for short and medium/long terms, respectively. Right: images of the sources from the combined 2009-2010 MERLIN data. Contour levels are drawn at $-1,1,1.414,2,2.828,4,5.656,8,11.282,16,22.564,32$, and $45.1286 \times 99 \mu \mathrm{Jy} / \mathrm{beam}$. The beam size $(50 \times 50 \mathrm{mas})$ is shown in the lower left-hand corner of each image.

\section{SN2008iz}

The light curve of SN2008iz derived from our 2009-2010 MERLIN/e-MERLIN data was extended back in time using Urumqi single-dish observations acquired in 2008-2009 [ []]. This light

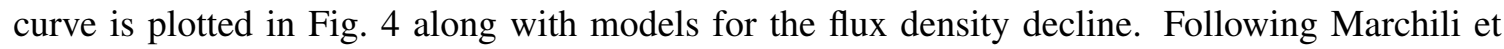
al. [प], we modelled this decline using an exponential function of the form

$$
S(t)=K_{1}\left(t-t_{0}\right)^{\beta} e^{-\tau} \quad \tau=K_{2}\left(t-t_{0}\right)^{\delta}
$$

where $t_{0}$ is the explosion date, a value of which (February 18, 2008) was recently estimated [ $[0]$.

As shown in Fig. 田, the 2009-2010 data, however, show a flattening in the light curve which is not properly accounted for by an exponential model. A power-law decay of the form

$$
S(t) \propto\left(t-t_{0}\right)^{\alpha}
$$




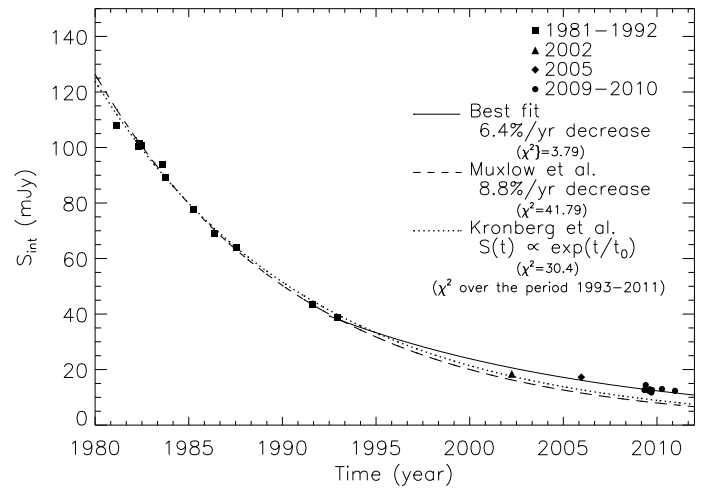

Figure 3: Light curve for $41.95+57.5$ over the period 1981-2010. Data are as follows: 1981-1991 from Kronberg et al. [目], 2002 from Fenech et al. [四], 2005 from Argo [四], 2009-2010 from this work. Models for the flux density decrease are based on an exponential decay [目] (dotted line), a $8.8 \% / y r$ decay [ [ ] (dashed line) and our best fit (6.4\%/yr decay, solid line) for the period 1993 2010. The values of $\chi^{2}$ quoted here correspond to the reduced chi-square statistics for each fit.

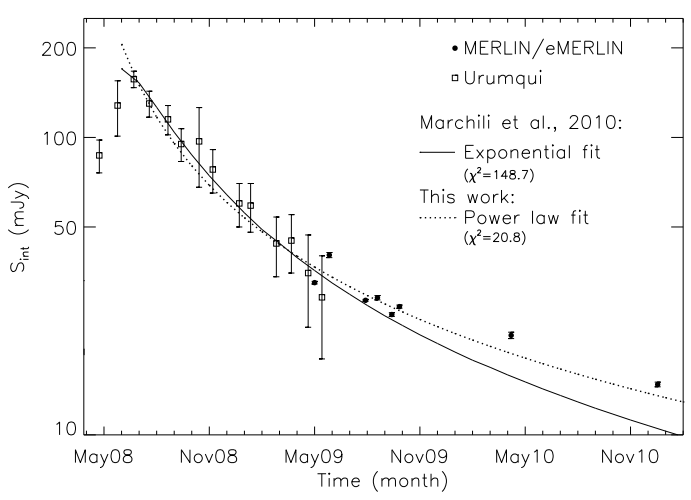

Figure 4: Light curve for SN2008iz. Data are from Urumqi single-dish [ [ $]$ (open squares) and MERLIN/ $e$-MERLIN (filled circles). An exponential decay model (solid curve) was fitted to the light curve using parameters from Marchili et al. [四, as well as a power-law decay model (dotted curve) displaying the best fit to the decay part of the light curve. The values of $\chi^{2}$ quoted here correspond to the reduced chi-square statistics for each fit.

with $\alpha=-1.15 \pm 0.05$, appears to yield a better fit (reduced chi-square of 20.8 vs 148.7 for the exponential fit). Whilst further measurements are required to confirm these results, there is tentative evidence that the rate at which the flux density of SN2008iz declines is becoming smaller.

\section{44.28+61.1 - A newly-detected SNR shell}

In order to search for possible new sources, each of the 51 known sources in M82 [田] was imaged and subtracted from the $u$ - $v$ data. The resulting dataset was then re-imaged. Following inspection of this residual image, one new source was identified. This source, located at position 09h55m53.00s, $+69^{\circ} 40^{\prime} 47.24^{\prime \prime}$ (J2000), was not visible in the 2002 MERLIN data. It has a peak flux density of $0.18 \mathrm{mJy} / \mathrm{beam}$ and an integrated flux density of $0.40 \pm 0.36 \mathrm{mJy}$. A shown in Fig. [1, it is shell-like in shape and is most likely a supernova remnant. By measuring the source extent, the age of the SNR may be inferred. The radius of the source was determined to be $140 \pm 5$ mas. At the distance of M82 (3.2 Mpc), this yields an estimated size of $2.1 \mathrm{pc}$. Fenech et al. [田] estimates that expansion velocities of SNRs in M82 are between 2200 and $10500 \mathrm{~km} / \mathrm{s}$, with a mean velocity of $5650 \mathrm{~km} / \mathrm{s}$. Using the latter value, we estimate that $44.28+61.1$ is approximately 360 years old.

\section{Conclusion}

We presented results of a monitoring campaign conducted on the galaxy M82 in 2009-2010 using MERLIN and $e$-MERLIN at 5 and $6 \mathrm{GHz}$, respectively. The data include eight epochs between May 2009 and December 2010. Light curves for all sources have been compiled, including flux density measurements from 2002 [四], 2005 [龱], and these new 2009-2010 monitoring data. We 


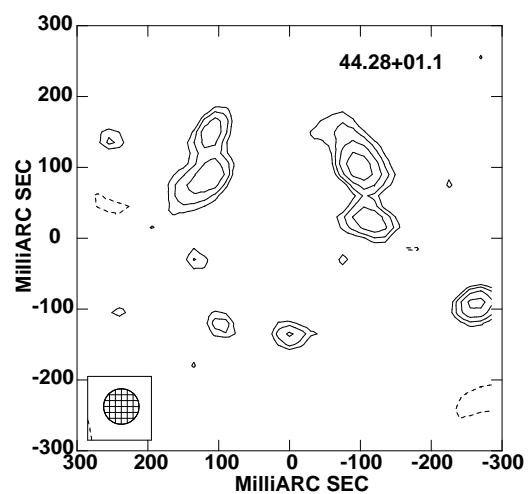

Figure 5: Image of 44.28+61.1. Contour levels are drawn at $-1,1,1.414,2,2.828,4,5.656,8$, and 11.282 $\times 99 \mu \mathrm{Jy} /$ beam. The beam size $(50 \times 50 \mathrm{mas})$ is shown in the lower left-hand corner of the plot.

have found that, in most of the sample, flux densities of SNRs and HII regions in M82 stay relatively constant, in agreement with previous analysis [6]. Aside from SN2008iz and the well-known variable source $41.95+57.5$, two additional sources, $43.18+58.2$ and 44.01+59.6, show signs of changes in flux density in the short and medium term. These sources are among the most compact SNRs in M82, possibly indicating that flux density variations are due to changes in the circumstellar and interstellar medium in which the shocks travel. They could hence be transitioning from supernovae to supernovae remnants, in analogy with recent results from Batejat et al. [వ] for Arp220. Our data also revealed a new source, $44.28+61.1$, which is most likely an SNR due to its shell-like shape.

\section{Acknowledgments}

M. A. Gendre would like to thank the organizers of the conference for providing financial support (through the RadioNet3 programme) to attend the symposium and present this paper.

\section{References}

[1] Argo, M. K., 2006, Ph.D. Thesis, Univ. Manchester, UK

[2] Batejat, F., Conway, J. E., Hurley, R., Parra, R., Diamond, P. J., Lonsdale, C. J., Lonsdale, C. J., 2011, ApJ, 740, id. 95 (14 pp.)

[3] Bietenholz, M. F., Bartel, N., Milisavljevic, D., Fesen, R. A., Challis, P., Kirshner, R. P., 2010, MNRAS, 409, 1594-1600

[4] Fenech, D. M., Muxlow, T. W. B., Beswick, R. J., Pedlar, A., Argo, M. K., 2008, MNRAS, 391, $1384-1402$

[5] Fenech, D., Beswick, R., Muxlow, T. W. B., Pedlar, A., Argo, M. K., 2010, MNRAS, 408, 607-621

[6] Kronberg, P. P., Sramek, R. A., Birk, G. T., Dufton, Q. W., Clarke, T. E., Allen, M. L., 2000, ApJ, 535, 706-711

[7] Marchili, N., Martí-Vidal, I., Brunthaler, A., Krichbaum, T. P., Müller, P., Liu, X., Song, H.-G., Bach, U., Beswick, R., Zensus, J. A., 2010, A\&A, 509, A47 (6 pp.)

[8] Muxlow, T. W. B., Pedlar, A., Beswick, R. J., Argo, M. K., O’Brien, T. J., Fenech, D., Trotman, W., 2005, Mem. S. A. It., 76, 586-588

[9] Trotman, W. M., 1996, M. Sc. Thesis, Univ. Manchester, UK 